\title{
Caracterización Anticorrosiva y de Biocompatibilidad en Recubrimientos Silicio-Titanio-Zirconio Sintetizados Vía Sol- gel y Depositados sobre Sustratos 316L
}

\author{
Jorge Bautista-Ruiz \\ Universidad Francisco de Paula Santander, Departamento de Física, Av. Gran Colombia 12E-96, San José de \\ Cúcuta - Colombia (e-mail: jorgebautista@ufps.edu.co)
}

Recibido Jun. 2, 2017; Aceptado Ago. 16, 2017; Versión final Sep. 25, 2017, Publicado Feb. 2018

\begin{abstract}
Resumen
Se sintetizaron, los sistemas ternarios [Si/Ti/Zr: 10/70/20] y [Si/Ti/Zr: 10/20/70] vía sol-gel y se aplicaron sobre sustratos de acero inoxidable $316 \mathrm{~L}$ mediante la técnica de inmersión. En los recubrimientos obtenidos se evaluó la respuesta anticorrosiva en una solución fisiológica simulada mediante curvas potenciodinámicas de polarización. Se estudió la biocompatibilidad celular de osteoblastos; la adhesión sustrato-recubrimiento; la topografía superficial, el espesor y la rugosidad en los recubrimientos. Los resultados de este estudio permiten establecer un alto grado de efectividad anticorrosiva y buena biocompatibilidad. Los recubrimientos sintetizados vía sol-gel establecen su potencialidad como barrera anticorrosiva en función del sistema ternario aplicado al sustrato de acero inoxidable 316L.
\end{abstract}

Palabras clave: propiedades anticorrosivas, sol-gel, silicio-titanio-zirconio, biocompatibilidad, adhesión

\section{Anticorrosive and Biocompatibility Characterization of Silicon- Titanium-Zirconium Coatings Synthesized by Sol-gel and Deposited on Substrates 316L}

\begin{abstract}
The ternary systems [Si/Ti/Zr: 10/70/20] and [Si/Ti/Zr: 10/20/70] were synthesized via sol-gel and applied on $316 \mathrm{~L}$ stainless steel substrates by dip-coating. In the coatings obtained, the corrosion response was evaluated in a simulated physiological solution using potentio-dynamic polarization curves. The cellular biocompatibility of osteoblasts was studied; Substrate-coating adhesion, and the surface topography and the roughness in the coatings. The results of this study allow establishing a high degree of anticorrosive effectiveness and good biocompatibility. Sol-gel uncoated coatings establish their potential as an anticorrosive barrier as a function of the ternary system applied to the $316 \mathrm{~L}$ stainless steel substrate.
\end{abstract}

Keywords: anticorrosive properties, sol-gel, silicon-titanium-zirconium, biocompatibility, adhesion 


\section{INTRODUCCIÓN}

Aunque la metodología sol-gel inició su desarrollo a mediados del siglo IXX con investigaciones desarrolladas por Pierre (1988) para obtener vidrios a temperatura ambiente en la actualidad ha evolucionado hasta el punto en el que se aplica a un amplio rango de estudios desde la obtención de recubrimientos, monolitos, material particulado a escala nanométrica, fibras, entre otras. Esta actividad científica creciente, permite establecer que la metodología sol-gel seguirá teniendo vigencia en el desarrollo de materiales especialmente en el campo de los recubrimientos (Pierre, 1988), (Fuentes et al., 2006). Específicamente, en la obtención de películas, el método sol-gel presenta ventajas sobre otras técnicas como son: (a) procesamiento a temperaturas relativamente bajas cuyo beneficio es el ahorro energético y disminución en el impacto ambiental, (b) diseño de productos con buena homogéneos y alta pureza (c) aplicación sobre sustratos en geometrías complejas, (d) posibilidad en el control de la composición química y la microestructura del producto final, y (e) equipamiento de bajo costo (Brinker y Scherer, 1990).

En la producción de películas vía sol-gel se involucra la evolución de redes inorgánicas a través de la formación de una suspensión coloidal (sol), y la gelación (envejecimiento) del sol para formar una red porosa en fase líquida, procesos que ocurren a partir de un compuestos organometálico (alcóxidos metálicos), los cuales se hidrolizan para obtener el sol y su posterior condensación a estado gel (Brinker y Scherer, 1990), (Sandoval, 2004). Después de obtener el sol, homogéneo y estable, es necesario establecer el tipo de técnica de conformado de los recubrimientos. Entre las técnicas con amplia aplicación están: electrodeposición, electroforesis, sedimentación, termoforesis, dispersión por centrifugación (spin coating), inmersión-extracción (dip coating), entre otras. En este desarrollo de este trabajo, se empleó la técnica dipcoating, y las condiciones experimentales establecidas en la conformación de recubrimientos cerámicos ternarios de $\mathrm{SiO}_{2}-\mathrm{TiO}_{2}-\mathrm{ZrO}_{2}$ propuestas por (Bautista-Ruiz, 2010).

Un biomaterial es cualquier material no biológico que reemplace la función de los tejidos o de los órganos vivos cuando son implantados (Sánchez y Campos, 2010). En el desarrollo de dispositivos para implantes, la naturaleza química y la textura superficial de los biomateriales son elementos determinantes en la biocompatibilidad, al entrar en contacto con el tejido vivo al introducirse en el cuerpo (Olivares et al., 2011) promoviendo una variedad de respuestas biológicas relacionadas con la adsorción de proteínas, la formación de trombos e inflamación; determinadas, por las propiedades superficiales de los materiales (Kang y Lee, 2012), (Garzón et al., 2013)

El acero inoxidable 316L, es utilizado en implantes ortopédicos, prótesis y stents por sus buenas propiedades de resistencia mecánica y de corrosión, pero no se recomienda emplearlo sin ningún recubrimiento o tratamiento superficial, porque puede producir una respuesta desfavorable para el organismo vivo, además de ser un material poco biofuncional. Caballero y colaboradores (2017), estudiaron la biocompatibilidad de $\mathrm{Ti}_{6} \mathrm{Al}_{4} \mathrm{~V}$ y acero inoxidable $316 \mathrm{~L}$ y la influencia del $\mathrm{pH}(4,5-6$ 7,5-10-12) en el comportamiento corrosivo, mediante Polarización Potenciodinámica Cíclica. Los resultados obtenidos indicaron que las condiciones de $\mathrm{pH} \mathrm{4,5}$ tienen un efecto negativo en la velocidad de corrosión de ambos materiales comparado con los otros valores $\mathrm{pH}$.

Se han desarrollado investigaciones en la cual utilizan la técnica de sol-gel para recubrir sustratos de titanio con diferentes óxidos $\left(\mathrm{Al}_{2} \mathrm{O}_{3}, \mathrm{Nb}_{2} \mathrm{O}_{6}, \mathrm{Ta}_{2} \mathrm{O}_{6}, \mathrm{TiO}_{2}, \mathrm{ZrO}_{2}\right)$ y luego compararon la viabilidad celular, in vitro, de células preosteoblásticas; concluyendo que la mejor biocompatibilidad la ofrece el recubrimiento de $\mathrm{Nb}_{2} \mathrm{O}_{6}$. También se ha estudiado la citotoxicidad y la adhesión de células humanas de osteosarcoma en $\mathrm{Ti}_{6} \mathrm{Al}{ }_{4} \mathrm{~V}$ superficialmente modificado empleando la prueba colorimétrica de viabilidad celular MTT, encontraron que las superficies tratadas no presentan citotoxicidad aparente. Otras investigaciones analizaron los efectos que tienen los tratamientos superficiales sobre la aleación de titanio $\mathrm{Ti}_{6} \mathrm{Al}_{4} \mathrm{~V}$ respecto al aumento de su biocompatibilidad, utilizando cultivos de células endoteliales de vena umbilical humana (Garzón et al., 2013).

En esta investigación, se seleccionó como material de soporte de las películas del sistema ternario sustratos de acero inoxidable AISI 316L. Es de resaltar que los aceros inoxidables tienen un amplio campo de aplicación en la industria y el campo de la salud en las cuales la efectividad anticorrosiva es de vital importancia (Cheraghia et al., 2012), (Sidanea et al., 2015), (Salahinejad et al., 2013), (Olivares, 2011), (De Mello y Balsamo, 2006) pero sufren procesos de corrosión por picadura al encontrarse en contacto son soluciones compuestas por iones $\mathrm{Cl}^{-}$. La protección superficial de materiales mediante recubrimientos cerámicos puede ser la solución de muchos problemas prácticos, especialmente cuando las condiciones de trabajo son adversas (temperaturas elevadas, ambientes químicamente agresivos, etc.) y en los cuales el reemplazo por otro tipo de materiales acarrea altos costos económicos. En salud, la aplicación del acero AISI 316L es muy importante en implantología quirúrgica, por su bajo costo en comparación con otros materiales como el titanio (Juan-Díaz et al., 2016), (Guo et al., 2015), (Xin et al., 2016). Los implantes de acero son considerados como un problema en salud pública. El acero cuando ha sido implantado en el 
cuerpo humano está sometido a ambientes corrosivos establecidos por las condiciones propias de los fluidos fisiológicos en contacto. Al sufrir ataque corrosivo el hierro de difunde en el cuerpo. El exceso de hierro en el organismo puede provocar hemosiderosis (un aumento de hierro en el tejido sin alteración de su estructura o función), o hemocromatosis (en la cual se daña el tejido) (Sugama, 2005).

El objetivo del presente trabajo fue depositar películas cerámicas del sistema ternario $\mathrm{SiO}_{2}-\mathrm{TiO}_{2}-\mathrm{ZrO}_{2}$, sobre sustratos de acero inoxidable 316L, en concentración de los precursores de los óxidos [Si/Ti/Zr: 10/70/20] y [Si/Ti/Zr: 10/20/70] mediante el método Sol gel a partir de Tetrabutóxido de titanio, Tetrabutóxido de zirconio y Tetraetil-ortosilicato como precursores. Se evaluó: la respuesta anticorrosiva en una solución fisiológica simulada, solución de Ringer, empleando Curvas Potenciodinámicas de Polarización; b) la topografía mediante Microscopía de Fuerza atómica - AFM, y c) biocompatibilidad. Los resultados se contrastaron con los obtenidos en probetas de acero sin recubrir, en función de la concentración de los precursores. Para complementar la investigación se decidió estudiar, adicionalmente, la adhesión, el espesor y la rugosidad de las películas. Se encontró que la efectividad biocompatible y anticorrosiva de los recubrimientos está relacionada directamente con la concentración de los precursores del sistema ternario que conforman el recubrimiento.

\section{MATERIALES Y MÈTODOS}

El procedimiento desarrollado para la conformación de los soles fue el siguiente (Bautista-Ruiz, 2010): Inicialmente se prepararon dos soluciones separadas, la primera de ellas contenía la mitad del volumen del solvente, etanol (EtOH), el volumen total del acomplejante (2,4 pentanodiona), el volumen total de precursor de titanio, tetrabutóxido de titanio $\left[\mathrm{Ti}(\mathrm{OBu})_{4}\right]$ y el volumen total del precursor de zirconio, tetrabutóxido de zirconio $\left[\mathrm{Zr}\left(\mathrm{OC}_{3} \mathrm{H}_{7}\right)_{4}\right]$. La segunda solución contenía la mitad del volumen de etanol, el volumen total del precursor de silicio, tetraetil-ortosilicato $\left[\mathrm{Si}\left(\mathrm{OC}_{2} \mathrm{H}_{5}\right)_{4}\right]$ y la mitad del volumen de agua. Las dos soluciones se mezclaron teniendo en cuenta que el alcohol cumple el papel de solvente y el agua da inicio al proceso de pre-hidrólisis del $\mathrm{Si}\left(\mathrm{OC}_{2} \mathrm{H}_{5}\right)_{4}$. Para obtener buena homogeneización de las soluciones, estas se mezclaron utilizando agitación a 400 r.p.m. a temperatura de $25^{\circ} \mathrm{C}$ y tiempo de dos horas. Los sustratos utilizados para depositar el sistema multicomponente, son chapas de acero AISI/SAE 316L de dimensiones $2 \mathrm{~cm} \mathrm{x} 2 \mathrm{~cm} \times$ $0,4 \mathrm{~cm}$, pulidas a brillo metalográfico, desengrasadas por 15 minutos empleando un equipo de ultrasonido con acetona y secadas en horno. Las películas se conforman por dip-coating a una velocidad de extracción de $13,2 \mathrm{~cm} / \mathrm{min}$.

La sinterización de las películas se efectúa a una velocidad de calentamiento de $1^{\circ} \mathrm{C} / \mathrm{min}$ permitiendo la eliminación controlada de los componentes orgánicos presentes en las películas. El proceso térmico se establece desde temperatura inicial de $25^{\circ} \mathrm{C}$ hasta $300^{\circ} \mathrm{C}$ y se estabiliza a ésta temperatura por una hora, posteriormente, se reanuda el calentamiento hasta $400^{\circ} \mathrm{C}$ y por media hora se equilibra. Finalmente, se llevan a temperatura ambiente a la velocidad de enfriamiento del horno. Los ensayos de curvas potenciodinámicas de polarización se llevan a cabo en una celda de tres electrodos, un electrodo plata/cloruro de plata $(\mathrm{Ag} / \mathrm{AgCl})$ como electrodo de referencia, de platino como contra-electrodo; y de trabajo, la muestra a analizar con área de exposición de $1 \mathrm{~cm}^{2}$. Para el análisis correspondiente se establecen los siguientes parámetros: velocidad de barrido de $1 \mathrm{mV} / \mathrm{s}$ con inmersión en el electrolito de solución de Ringer; intervalo de potencial desde $-0,200 \mathrm{mV}$ hasta $200 \mathrm{mV}$. Se determinan las velocidades y los potenciales de corrosión mediante extrapolación de Tafel empleando un potenciostato-galvanostato marca GAMRY Instruments; resolución de voltaje de $20 \mathrm{~V}$ y de corriente de $1 \mathrm{fA}$. El tiempo de inmersión antes de realizar la prueba fue de 45 minutos. Para cada recubrimiento se ejecutan tres pruebas potenciodinámicas. Los valores de potencial de corrosión ( $E_{\text {corr }}$ ) y de densidad de corriente de corrosión ( $i_{c o r r}$ ) se determinan por el método de extrapolación Tafel mediante el software Gamry Echem Analyst versión 5.3.

Para la evaluación topográfica de los recubrimientos se emplea un equipo AFM NaioAFM Nanosurf en modo contacto. Las pruebas de biocompatibilidad se desarrollan por medio de donadores sanguíneos con edad promedio de 25 a 35 años, cada una de las muestras se le agrega anticoagulante y se centrifugan por un tiempo de 5 minutos a 5000 rpm. En el ensayo de adhesión, las células son sembradas e incubadas por 24 horas. Luego de la incubación, las células sin adherir al sustrato son retiradas mediante lavados con agua estéril. Las células que permanecen adheridas al sustrato son fijadas y teñidas con $300 \mu$ de una solución compuesta por $0.1 \%$ de azul de toluidina y $3.5 \%$ de paraformaldehido. Luego de 24 horas a temperatura ambiente, $100 \mu \mathrm{l}$ del sobrenadante son usados para medir la absorción óptica a 630 nm mediante un ensayo inmunoabsorbente ligado a enzimas, esto debido a que el número de células adheridas al sustrato es proporcional a la absorbancia. El otro ensayo es de proliferación celular, basado en la habilidad de la enzima mitocondrial succinato-deshidrogenasa, para la reducción metabólica del Bromuro de 3-(4,5dimetiltia-zol-2-ilo)-2,5-difeniltetrazol (MTT), esta reducción tiene como resultado un compuesto coloreado de color azul (formazán) lo cual determina la funcionalidad mitocondrial de las células tratadas. Este método 
se ha utilizado para medir la proliferación celular; en donde la cantidad de células vivas es proporcional a la cantidad de formazán producido. Las células se cultivan e incuban en tiempos de 24, 48, 120, 144 y 168 horas.

La evaluación de la adherencia de los recubrimientos al sustrato $316 \mathrm{~L}$ se desarrolla por medio de pruebas de rayado con un equipo CSM Revetest Xpress Scratch Tester. La carga es progresivamente aplicada a una velocidad de rayado de $10,11 \mathrm{~mm} / \mathrm{min}$ hasta un valor máximo de $20 \mathrm{~N}$, en una longitud de rayado de $6,4 \mathrm{~mm}$, y tasa de carga de $30 \mathrm{~N} / \mathrm{min}$, con un indentador Rockwell C de $200 \mu \mathrm{m}$ de radio. Para medir el espesor, de cada recubrimiento, se emplea un perfilómetro DEKTAK 150 con repetibilidad de $6 \AA$. Las mediciones se efectúan con barrido de $600 \mu \mathrm{m}$, con duración de $30 \mathrm{~s}$, aplicando una fuerza de $1 \mathrm{mg}$, con perfil de valles y crestas, y resolución de $0,067 \mu \mathrm{m} /$ muestra.

\section{RESULTADOS Y ANÁLISIS}

Se presentan los resultados de la evaluación de las películas del sistema ternario y del sustrato 316L analizando aspectos de corrosión, biocompatibilidad, topografía, adherencia, y espesores.

\section{Corrosión}

La gráfica de la figura 1 revela los diagramas de Tafel del sistema $\mathrm{Bi} / \mathrm{Ti} / \mathrm{Zr}$ en las dos concentraciones de estudio, y su comparación con respecto al sustrato 316L. Se observa la variación de los potenciales de corrosión de las películas cerámicas con respecto al potencial de corrosión del sustrato. En general, el potencial de corrosión tiende a tomar valores más positivos a medida que aumenta la concentración del compuesto titanio, comportamiento observado para películas [Si/Ti/Zr: 10/70/20] y corroborado al comparar los resultados de los valores del potencial de corrosión, y corriente de corrosión tanto como para los recubrimientos como para el sustrato, como muestra la tabla 1. Conocer la tendencia de los parámetros anteriormente mencionados permitirá conocer el comportamiento de la interfase sustrato-recubrimiento en cada caso. Se observa que todos los recubrimientos indican valores de potencial de corrosión más positivos que el valor encontrado para el sustrato de acero inoxidable 316L. Con respecto a las corrientes de corrosión, se indica que estos valores son menores con respecto a la información encontrada para el sustrato sin recubrir.

Tabla 1: Valores de corriente, potencial y velocidad de corrosión.

\begin{tabular}{|c|c|c|c|}
\hline Muestra & $\begin{array}{c}\text { Corriente de } \\
\text { corrosión }(n A)\end{array}$ & $\begin{array}{c}\text { Potencial de } \\
\text { corrosión }(m V)\end{array}$ & $\begin{array}{c}\text { Velocidad de corrosión } \\
(m p y)\end{array}$ \\
\hline$[\mathrm{Si} / \mathrm{Ti} / \mathrm{Zr}: 10 / 20 / 70]$ & 156.01 & -150.10 & $394.2 \times 10^{-3}$ \\
\hline$[\mathrm{Si} / \mathrm{Ti} / \mathrm{Zr}: 10 / 70 / 20]$ & 52.90 & -122.20 & $133.5 \times 10^{-3}$ \\
\hline $\mathrm{AISI} 316 \mathrm{~L}$ & 601.10 & -192.10 & 106.4 \\
\hline
\end{tabular}

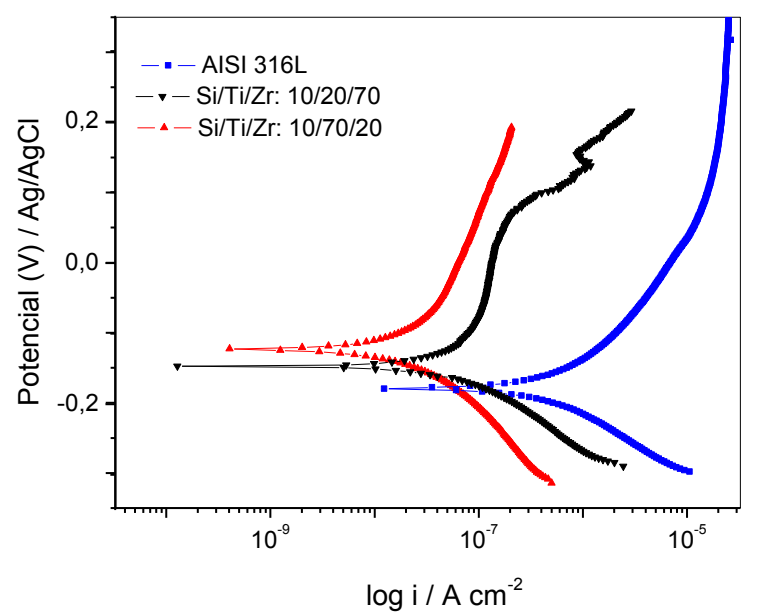

Fig. 1: Curvas potenciodinámicas de polarización para los recubrimientos $\mathrm{Si} / \mathrm{Ti} / \mathrm{Zr}$ y el sustrato $316 \mathrm{~L}$

Se evidenció que la aplicación de las películas cerámicas al sustrato fue óptima. Las temperaturas y la velocidad de calentamiento en el proceso de sinterizado establecido, no favorece el surgimiento de gran cantidad de poros. También se evidencia de los resultados, que los coeficientes de dilatación térmica, 
película y sustrato, no son tan diferentes y por tanto no asiste al surgimiento de grietas en la película que permitiera el paso de iones presentes en la solución de trabajo (Ringer).

Con respecto a la velocidad de corrosión tanto de las películas como del sustrato, los datos registrados en la tabla 1, permiten inferir que la diferencia es de tres órdenes de magnitud, estableciendo que los recubrimientos actúan como muy buena barrera anticorrosiva. Se determinó al comparar los resultados de las velocidades de corrosión entre las películas y el sustrato una disminución de este parámetro en $99 \%$ tanto para películas [Bi/Ti/Zr: 10/70/20] como para recubrimientos [Bi/Ti/Zr: 10/20/70]. Comparado los valores de la velocidad de corrosión entre las películas estudiadas se encontró que en los recubrimientos [Bi/Ti/Zr: 10/70/20] la disminución es del $66 \%$ con respecto a las capas [Bi/Ti/Zr: 10/20/70] cuando se emplea solución de Ringer como electrolito.

Continuando con el análisis de los resultados, se establece el porcentaje de efectividad protectora $E_{f}(\%)$ (Chang, Chung, y Lai, 2004), (Altun y Sen, 2005), (Moreno, Hernández, Santana, y et.al., 2012), (Yoo, Le, Kim, y et.al., 2008) de los recubrimientos del sistema Si-Ti-Zr en función de la concentración de los precursores mediante la expresión (1):

$E f(\%)=\left(\frac{I_{\text {corr }_{s}}-I_{\text {corrt }}}{I_{\text {cors }}}\right) \times 100$

donde Icorr $_{s}$ es la densidad de corriente de corrosión del sustrato sin recubrir e Icorr $r_{f}$ es la densidad de corriente de corrosión del sustrato recubierto. Se encontró que la efectividad anticorrosiva para películas [Si/Ti/Zr: 10/70/20] es de $91 \%$ y del $74 \%$ para recubrimientos [Si/Ti/Zr: 10/20/70].

\section{Biocompatibilidad}

La figura 2 muestra las curvas de citotoxicidad en cada uno de los recubrimientos. Se observa que el recubrimiento con mayor actividad celular es el [Si/Ti/Zr: 10/70/20] con un aumento con respecto al sustrato del $121 \%$ seguido del [Si/Ti/Zr: 10/20/70] con un aumento en el número de células del 110\%. Entre películas la proliferación celular está en $5 \%$. Según la figura 2 y lo mencionado anteriormente, se infiere que los niveles de absorbancia establecidos para las películas son superiores a los encontrados en el acero inoxidable AISI 316L. En la figura 3 se observa la diferencia existente entre los niveles de adhesión celular en células de osteoblastos en los recubrimientos y el sustrato. Al comparar los resultados según la concentración de los recubrimientos, no se observa gran diferencia entre ellas. Sin embargo, el recubrimiento [Si/Ti/Zr: 10/70/20] presenta mayores niveles de adhesión celular y en general se demuestra una diferencia significativa entre los resultados obtenidos para los recubrimientos y el acero inoxidable AISI 316 L. Se estableció en un $90 \%$ el índice de confiabilidad de la adhesión celular mediante el Test de Bonferroni.

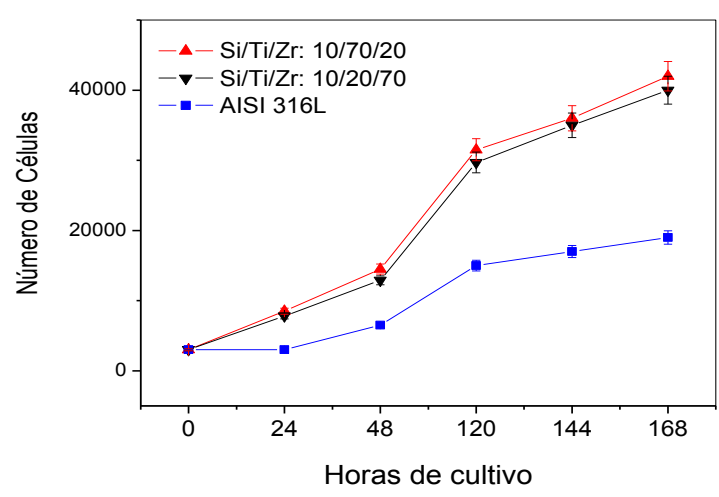

Fig. 2: Curvas de citotoxicidad para los recubrimientos del sistema $\mathrm{Si} / \mathrm{Ti} / \mathrm{Zr}$ y el sustrato.

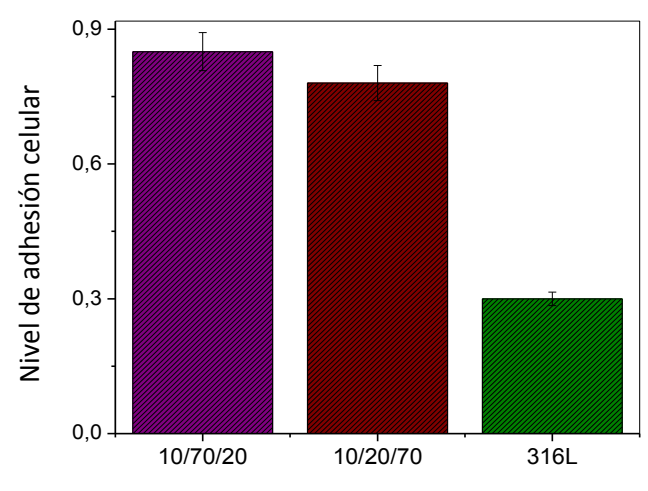

Fig. 3: Niveles de adhesión celular para las películas $\mathrm{Si} / \mathrm{Ti} / \mathrm{Zr}$ y el sustrato $316 \mathrm{~L}$

La figura 4 muestra las fotografías de las células adheridas a las superficies del sustrato y de los recubrimientos. Estas fotografías corroboran las tendencias indicadas en las figuras 2 y 3 . En términos generales, las células de osteoblastos presentan una adhesión celular directamente proporcional al periodo de incubación, este comportamiento establece que la composición de los recubrimientos no interfiere con los procesos de crecimiento celular. Las diferencias en los parámetros para cada uno de los recubrimientos acelera el crecimiento celular, estas condiciones ambientales favorecen los procesos celulares permitiendo un adecuado crecimiento. Se puede concluir, que los recubrimientos estudiados crean condiciones óptimas 
de crecimiento en comparación con los sustratos de acero AISI 316L sin recubrir. A 168 horas de cultivo, el incremento de la adhesión celular de las películas con respecto al sustrato se calcula en $183 \%$ para recubrimientos [Si/Ti/Zr: 10/70/20] y del 160\% para [Si/Ti/Zr: 10/20/70]. Al estimar, entre películas, la adhesión celular se encontró una variación de aproximadamente el $9 \%$.

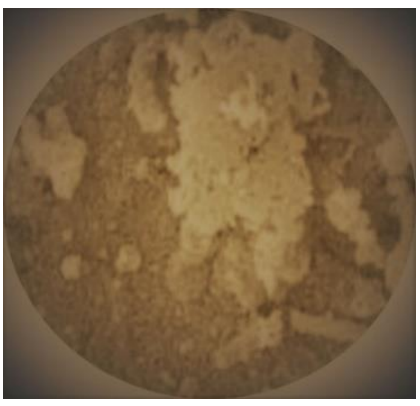

a)

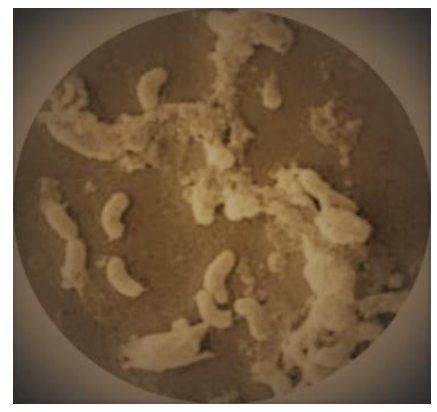

b)

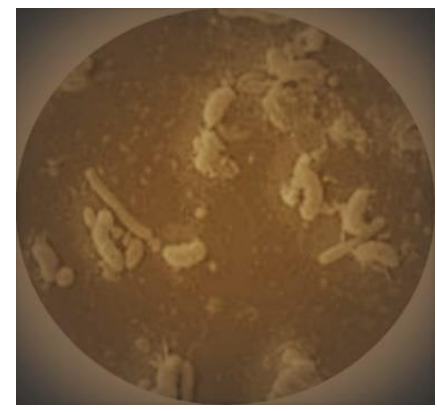

C)

Fig. 4: Detalles del comportamiento celular: a) [Si/Ti/Zr: 10/70/20], b) [Si/Ti/Zr: 10/20/70] y c) sustrato 316L.

Los comportamientos, desde el punto de vista de biocompatibilidad, descritos anteriormente se explican por la unión de las células de osteoblastos a la superficie del recubrimiento originado por fuerzas de Van der Waals, por la tensión superficial generada entre la superficie de las películas y las biomoléculas de integrina que generan puentes de hidrógeno, así se genera una unión proteica que es adsorbida en la superficie del recubrimiento. Las integrinas en la superficie de la película contribuyen a la organización tisular mediante su unión a moléculas de la matriz extracelular y de la membrana basal en la superficie del material. Los enlaces covalentes e iónicos son los responsables de la adhesión celular. El factor celular aporta a la unión biomolecular una capa monoatómica con los óxidos de la superficie y las capas moleculares más complejas.

\section{Topografía}

La figura 5 presenta las micrografías de AFM para los recubrimientos del sistema $\mathrm{Si} / \mathrm{Ti} / \mathrm{Zr}$ obtenidos variando la concentración de los precursores. Al comparar los comportamientos superficiales de las películas (figura 5 a) y b)) y el sustrato (figura 5 c)) no se observa una variación apreciable en la topografía. Los recubrimientos presentan superficies muy homogéneas y consolidadas con algunas imperfecciones propias del proceso de inmersión. En la figura $5 \mathrm{c}$ ) se observa la topografía del sustrato, evidenciando una superficie algo homogénea con algunas imperfecciones originadas en el proceso de pulido y acondicionamiento para soportar los recubrimientos. Además de evaluar la topografía, la técnica AFM permitió determinar los valores de rugosidad superficial de cada una de las muestras estudiadas.

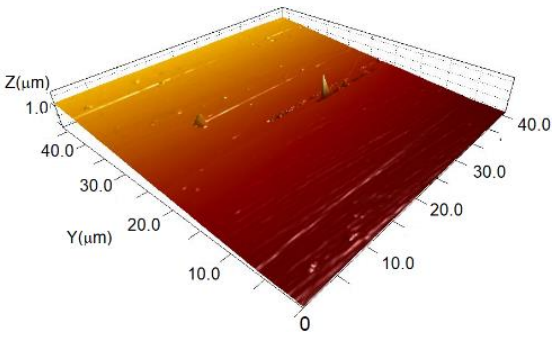

a)

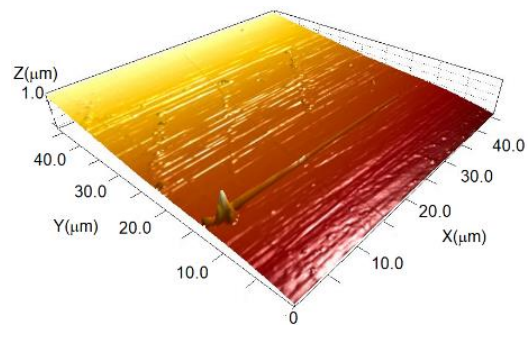

b)

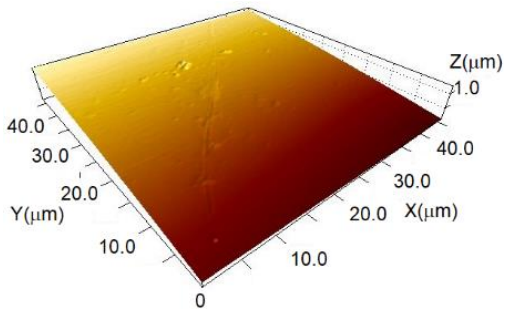

c)

Fig. 5: Imágenes de AFM para los recubrimientos y el sustrato: a) [Si/Ti/Zr: 10/70/20], b) [Si/Ti/Zr: 10/20/70] y c) 316L.

La técnica AFM permitió cuantificar la rugosidad mediante el registro de perfiles de alturas, con lo cual se obtuvieron parámetros como la rugosidad $\mathrm{P}_{\text {sa }}$ y la rugosidad promedio $\mathrm{P}_{\mathrm{a}}$. La Tabla 2, resume los valores de rugosidad para cada una de las películas estudiadas y el sustrato $316 \mathrm{~L}$.

Tabla 2: Valores de rugosidad para las películas $\mathrm{Si} / \mathrm{Ti} / \mathrm{Zr}$ y el sustrato $316 \mathrm{~L}$.

\begin{tabular}{|c|c|c|}
\hline Muestra & $P s a(\mu \mathrm{m})$ & $P a(\mu \mathrm{m})$ \\
\hline$[\mathrm{Si} / \mathrm{Ti} / \mathrm{Zr}: 10 / 20 / 70]$ & 0.221 & 0.247 \\
\hline$[\mathrm{Si} / \mathrm{Ti} / \mathrm{Zr}: 10 / 70 / 20]$ & 0.184 & 0.185 \\
\hline 316L & 0.160 & 0.137 \\
\hline
\end{tabular}


Según los resultados de la tabla 2, se observa un aumento de la rugosidad $(\mathrm{Pa})$ en las películas con respecto al sustrato AISI 316L. Para el caso de las películas [Si/Ti/Zr: 10/70/20], el valor de la rugosidad disminuye y está sobre los valores registrados para el sustrato. El aumento del valor de la rugosidad se explica porque para los cálculos de $P a$ es el promedio aritmético de los valores absolutos de las alturas $y(x)$ medidas a partir de una línea central. Matemáticamente Ra, lo define Hinojosa (Hinojosa y Reyes, 2001) como se muestra en la ecuación 2 , en la que $L$ es la longitud de muestreo.

$R_{a}=\frac{1}{L} \int_{0}^{L}|y(x)| d x$

Si se descarta la contribución de los valores de la rugosidad de las estrías pseudo-períodicas, es posible establecer que una cantidad mayor de la concentración del precursor de titanio en las películas homogeniza más la superficie. Pero también influye en estos resultados el proceso de sinterizado, al establecer una velocidad de calentamiento lenta para evitar la generación de poros o grietas. En cuanto a la superficie del material, el grado de rugosidad es ventajosa desde el punto de vista mecánico porque ofrece una adecuada distribución de las tensiones a escala celular conformando un sistema retentivo que evita el micromovimiento lo que comprometería la interfase celular.

\section{Adherencia}

El estudio de adherencia consistió en analizar la huella sobre los recubrimientos del sistema $\mathrm{Si} / \mathrm{Ti} / \mathrm{Zr}$, evidenciando fallas como: la existencia y proliferación de grietas, el cuarteamiento de los recubrimientos, su desprendimiento total o parcial y el hundimiento de la superficie alrededor de la huella, como se ve en la Fig. 6.

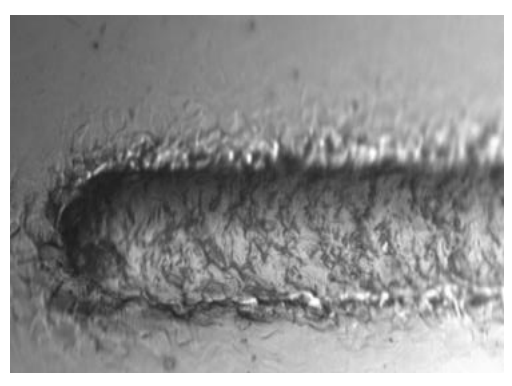

a)

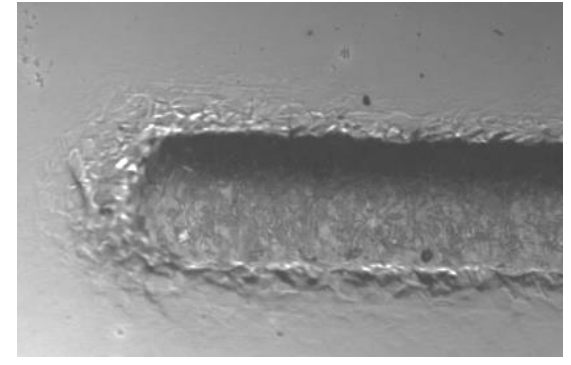

b)

Fig. 6: Huellas residuales de la prueba de rayado de los recubrimientos: a) [Si/Ti/Zr: 10/20/70] y b) [Si/Ti/Zr: 10/70/20]

La figura 6 muestra los resultados de la huella de adherencia en la última zona de la prueba de adhesión para los recubrimientos $\mathrm{Si} / \mathrm{Ti} / \mathrm{Zr}$. En general, no se observaron cambios significativos en los valores de carga crítica con respecto a la concentración de los recubrimientos, que oscilaron entre 10 y $12 \mathrm{~N}$. En esta figura (6) se observa que los dos tipos de recubrimientos presentan el mismo mecanismo de fractura bajo una carga que aumenta en forma lineal y a velocidad constante en todo el recorrido del indentador. También se evidencia, en zonas contiguas a la huella, modificaciones en la textura del recubrimiento relacionadas a la deformación plástica. En regiones intermedias de la huella de desgaste se originan grietas con escaso cuarteamiento del recubrimiento, que posiblemente generan pérdida de adherencia del sistema.

Este comportamiento se puede explicar por la buena adhesión de las películas en función de la similitud de los coeficientes de dilatación térmica entre el sustrato $316 \mathrm{~L}$ y los recubrimientos. Esta analogía permite establecer la importancia del adecuado proceso de sinterizado, evitando la generación de grandes esfuerzos residuales de comprensión en las películas. La fuerza de adhesión del sistema sustratorecubrimiento tiene su origen, por lo general, en los enlaces moleculares propios. Es importante resaltar que las fuerzas de unión entre dos superficies varían en magnitud según sea su origen: químico, electrostático, fuerzas de van der Waals, anclaje mecánico, capilaridad o combinaciones de éstas. Además de las fuerzas de adhesión, la estabilidad de la interfase sustrato-recubrimiento está condicionada por las tensiones residuales, como se mencionó anteriormente.

\section{Espesores}

Los resultados de la medición de los espesores para las películas del sistema $\mathrm{Si} / \mathrm{Ti} / \mathrm{Zr}$, permiten establecer valores de 390,80 nm para recubrimientos [Si/Ti/Zr: 10/70/20] y de 306, $42 \mathrm{~nm}$ para películas [Si/Ti/Zr: 10/20/70]. La diferencia, está relacionada con la concentración de los precursores en cada sistema ternario, debido a que las películas se obtuvieron a la misma velocidad de extracción al emplear la técnica dip-coating. 


\section{CONCLUSIONES}

De acuerdo a los resultados de este estudio, y de su discusión y análisis se pueden extraer las siguientes conclusiones principales:

1) La optimización de las condiciones de sinterización de las películas Si/Ti/Zr permitieron la obtención de películas densas con poca formación de microporos en función de la concentración de los precursores. Se determinó la influencia de las altas concentraciones de tetrabutóxido de titanio en las buenas características de densificado de los recubrimientos. 2) Al evaluar la adhesión en los recubrimientos se observa la aparición de las primeras fallas correlacionadas con la carga cohesiva. La segunda falla corresponde con el levantamiento o de laminación de la película y falla total del recubrimiento. 3) La caracterización anticorrosiva por curvas potenciodinámicas de polarización muestran que los recubrimientos con alto contenido de tetrabutóxido de titanio (precursor del titanio) mejoran la respuesta al ataque de corrosivo, con respecto al sustrato, cuando se encuentran inmersos en solución de Ringer. Se determinó un bajo porcentaje de porosidad y alto porcentaje de efectividad anticorrosiva en los recubrimientos dependiendo de su concentración. 4) Los recubrimientos muestran muy buena biocompatibiidad permitiendo una adecuada adhesión de las células a la superficie de las películas.

\section{REFERENCIAS}

Altun H. y S. Sen, The Effect of DC magnetron sputtering AIN coatings on the corrosion behaviour of magnesium alloys, Surf. Coat. Technol.: 197, 193-197 (2005)

Bautista-Ruiz, J., Efecto del número de capas en las propiedades superficiales de recubrimientos $\mathrm{Si} / \mathrm{Ti} / \mathrm{Zr}$ obtenidos a partir de suspensiones sol-gel, Revista Científica Guillermo de Ockham: 8, 141-148 (2010)

Brinker, C. y G. Scherer, Sol-gel Science: The Physics and Chemistry of Sol-gel Processing, 13-56, Academic Press, Boston, USA (1990)

Caballero, J.; E. Correa y H. Estupiñán, Análisis de la biocompatibilidad de $\mathrm{Ti}_{6} \mathrm{Al}_{4} \mathrm{~V}$ y acero inoxidable $316 \mathrm{LVM}$ basado en efectos de pH, aplicando criterios de la norma ASTM F2129, Ingeniare.: 25(1), 95-105 (2017)

Chang, K.L.; S.C. Chung y S. Lai, The electrochemical behavior of thermally oxidized CrN coatings deposited on steel by cathodic arc plasma deposition, Appl. Surf. Sci.: 236(6), 406-415 (2004)

Cheraghia, H.; M. Shahmiria y Sadeghianb, Corrosion behavior of $\mathrm{TiO}_{2}-\mathrm{NiO}$ nanocomposite thin films on AISI 316L stainless steel prepared by sol-gel method, Thin Solid Films: 522, 289-296 (2012)

De Mello, J. y P. Balsamo, Comportamiento Tribológico de Aceros Inoxidables para Cubertería, Inf. Tecnol.: 17(6), 57-62 (2006)

Fontana, M., Corrosion Engineering, 15-26, McGraw-Hill, Inc. Boston, USA (1987)

Fuentes, R.; J. Ramírez, M. Reyes y V. Castaño, Respuesta al Desgaste de un Material de Aluminio Reforzado con Circonia. Inf. Tecnol.: 17(3), 47-52 (2006)

Garzón, A.; N. Aguirre y J. Olaya, Estado del arte en biocompatibilidad de recubrimientos, Visión Electrónica, 7(1), 160-177 (2013)

Guo, L.; W. Feng, X. Liu y C. Lin, Sol-gel synthesis of antibacterial hybrid coatings on titanium, Materials Letters: 160(1), 448-451 (2015)

Hinojosa, M. y M. Reyes, La rugosidad de las superficies: Topografía, Ingeniería: 3, 27-33 (2001)

Juan-Díaz, M.; M. Martínez-lbáñez y S. Lara-Sáez, Development of hybrid sol-gel coatings for the improvement of metallic biomaterials performanc, Progress in Organic Coatings: 96, 42-51 (2016)

Kang, C. y Y. Lee, Carbohydrate polymer grafting on stainless steel surface and its biocompatibility study, Journal of industrial and Engineering Chemistry, 18(5), 1670-1675 (2012)

Moreno, C.; S. Hernández, J. Santana y otros tres autores, Characterization of Water Uptake by Organic Coatings Used for the Corrosion Protection of steel as Determined from Capacitance Measurements, Int. J. Electrochem. Sci.: 7, 8444-8457 (2012) 
Olivares, R., Biocompatibility of niobium Coatings, Coatings: 1, $72-87$ (2011)

Olivares, R.; J. Olaya, C. Ramírez y S. Rodil, Biocompatibility of niobium coatings, Coatings: 1, $72-87$ (2011)

Pierre, A., Introduction to sol-gel, 35-40, Academic Publishers, Boston, USA (1988)

Salahinejad, E.; M. Hadianfard y D. Macdonald, A new double-layer sol-gel coating to improve the corrosion resistance of a medical-grade stainless steel in a simulated body fluid, Materials Letters: 97, 162-165 (2013)

Sandoval, R.; J. Jiménez, R. García y A. Melo, Preparación Vía SolGel y Caracterización del Sistema $\mathrm{CoMo} / \mathrm{Al}_{2} \mathrm{O}_{3} \mathrm{TiO}_{2}$ para la Hidrodesulfuración de un Gasóleo Pesado de Vacío, Inf. Tecnol.: 15(3), 81-84 (2004)

Sidanea, D.; D. Chicotb, D. y S. Yalaa, Study of the mechanical behavior and corrosion resistance of hydroxyapatite sol-gel thin coatings on $316 \mathrm{~L}$ stainless steel pre-coated with titania film, Thin Solid Films: 593, 71-80 (2015)

Sugama, T., Cerium acetate-modified aminopropylsilane triol: A precursor of corrosion-preventing coating for aluminum-finned condensers, Journal of Coatings Technology and Research: 2(8), 649-659 (2005)

Xin, Z; M. Lei, W. Jian-gang y Z. Hui-min, Investigation on ultrathin titanium oxide films synthesized by surface sol-gel method, International Journal for Light and Electron Optic: 127(5), 2780-2783 (2016)

Yoo, Y.; D. Le, S. Kim y otros cuatro autores Corrosion Behavior of TiN, TiAIN, TiAISiN Thin Films Deposited on Tool Steel in the 3.5 wt.\% NaCl Solution, Thin Solid Films: 516, 3544-3548 (2008) 
